\title{
Effect of doping with Carbon and Boron Nitride on the Hydrogen absorption in Titanium
}

A.V. Postnikov, ${ }^{1}$ O.S. Morozova,${ }^{2}$ Ch. Borchers, ${ }^{3}$, E.Z. Kurmaev ${ }^{4}$ and A. Moewes ${ }^{5}$

${ }^{1}$ LPMD, Paul Verlaine University - Metz, 1 Bd Arago, F-57078 Metz, France

postnikov@univ-metz.fr

${ }^{2}$ Semenov Institute of Chemical Physics RAS, Kosygin st. 4, 119991 Moscow, Russia

${ }^{3}$ Institute for Materials Physics, University of Göttingen, Friedrich-Hund-Platz 1, D-37077 Göttingen, Germany

${ }^{4}$ Institute of Metal Physics RAS-Ural Div., Kowalevskaya st. 18, 620219 Yekaterinburg, Russia

${ }^{5}$ Department of Physics and Engineering Physics, University of Saskatchewan, 116 Science Place, Saskatoon, SK, S7N 5E2, Canada

Pure metallic titanium is a fairly good hydrogen-absorbing material, which permits to accommodate up to $\sim 3.3 \times 10^{-3} \mathrm{~mol} / \mathrm{g} \mathrm{Ti}$, or $\mathrm{H} / \mathrm{Ti}=0.32$. The reaction goes fast during reactive milling of $\mathrm{Ti}$ in the $\mathrm{H}_{2}$ atmosphere. The "theoretical limit" of the hydrogen sorption, with all octahedral interstitials occupied by hydrogen atoms, corresponds to the (cubic) titanium hydride $\mathrm{TiH}_{2}$. It is known ${ }^{1,2}$ that an addition of graphite considerably accelerates the hydrogen uptake in the process of milling. However, the carbonization of the surface ultimately blocks the $\mathrm{H}_{2}$ absorption. In the search for other additives which could boost the hydrogen uptake in a similar manner, the boron nitride, another layered material, turned out quite efficient. ${ }^{2}$ Even if the maximal sorption rate is inferior to that with the carbon additive, the sorption process is more stable and, in total, accommodates by far more hydrogen, notably in a post-milling absorption, ${ }^{2}$ shifting the total hydrogen sorption towards $\mathrm{H} / \mathrm{Ti}=0.2$ (after $30 \mathrm{~min}$. of milling), 0.88 (66 min.) and 1.95 (205 min.), the last number being close to the theoretical absorption limit.

The details of the microscopics of the hydrogen absorption are, however, not obvious. A combined study by X-ray diffraction, electron microscopy and X-ray emission spectroscopy (see details in Ref. 2) revealed the following, in part contradictory, findings:

1. The phase analysis by the X-ray diffraction shows that the amount of $\alpha$-Ti and hexagonal $\mathrm{BN}$ gradually decreases in the process of milling $\mathrm{H}_{2}$ absorption, leaving finally (after 206-min. milling) exclusively $\delta-\mathrm{TiH}_{1.95}$. No traces of $\mathrm{TiN}$ or $\mathrm{TiB}$ phases have been detected at any stage of the process.

2. According the X-ray emission spectroscopy, which probes the local electronic structure in an element-sensitive way, the $L_{2,3}$ spectrum of titanium in the milled hydrogen-saturated sample resembles a superposition $40 \% \mathrm{Ti}+40 \% \mathrm{TiH}_{2}+20 \% \mathrm{TiN}$. The $K_{\alpha}$ spectrum of nitrogen in the same sample can be rouphly decomposed as $89.7 \% \mathrm{BN}+10.3 \% \mathrm{TiN}$.

An apparent controversy (a noticeable amount of TiN according to XES, but no traces of the nitride phase in the diffraction study) reflects the fact that the X-ray spectroscopy is sensitive to the nearest chemical neighbourhood. A contribution of TiN-like spectrum reveals merely that nitrogen atoms exist either in the boron nitride layers (on the surface of titanium particles), or in the TiN-like neighbourhood, that is, octahedrally coordinated by titanium atoms. The latter situation corresponds to a nitrogen atom in an octahedral interstitial within hexagonal $(\alpha)$ or cubic $\left(\mathrm{TiH}_{2}\right.$-like) titanium lattice. Such a conclusion offers a foundation for a microscopic $a b$ initio study of the electronic structure and chemical bonding. Namely, we adopt a structural model which does not contain BN (in crystalline or molecular form inside the titanium bulk) but allows single nitrogen (and, for the sake of comparison, also boron and carbon) atoms in 


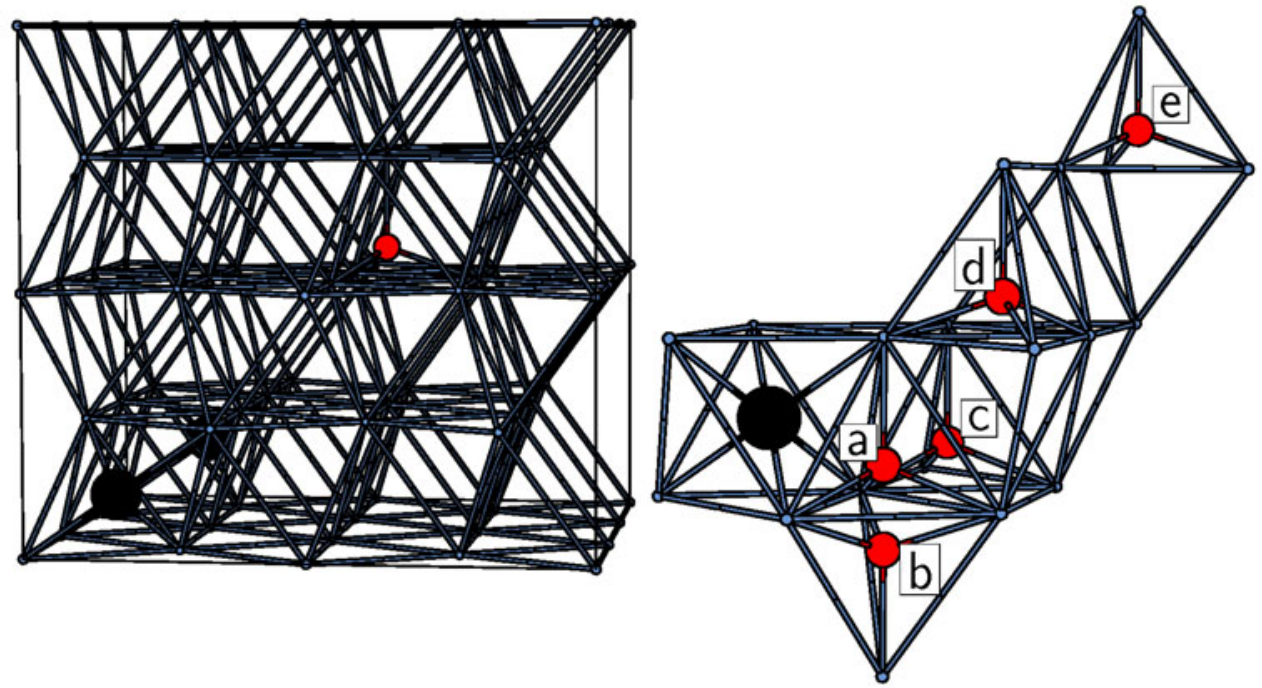

Figure 1: Left panel: a supercell of hcp-Ti with 48 atoms (shown as bond wires), with an interstitial B, N or C in the octa-pore (black sphere) and maximally distant tetra-interstitial hydrogen (light sphere). Right panel: possible tetra-interstitial sites of $\mathrm{H}$ relative to the octa-interstitial $2 p$ atom, considered in the calculation.

octahedral interstitials. A single hydrogen atom is placed in tetrahedral interstitials at different distances from the $2 p$ atom. Our primary objective was the study of the "interaction potential" between $\mathrm{H}$ and a $2 p$ atom in the crystal, or, put differently, how the hydrogen affinity of titanium is affected by the presence of interstitial $\mathrm{N}, \mathrm{B}$, or $\mathrm{C}$.

The calculations have been done within the density functional theory paradigm (using specifically local density approximation for the exchange-correlation), applying the method and computer code SIESTA ${ }^{3,4}$. Norm-conserving pseudopotentials have been generated for "nominal" valence charge configurations (B2 $2 s^{2} 2 p^{1}, \mathrm{C} 2 s^{2} 2 p^{2}, \mathrm{~N} 2 s^{2} 2 p^{3}$, Ti $4 s^{1} 3 p^{6} 3 d^{2}$ ) by the TroullierMartins scheme. ${ }^{5}$ Strictly confined basis functions were of the "double- $\zeta$ with polarization orbitals" quality. Calculations have been done on a supercell of $48 \mathrm{Ti}$ atoms, with one hydrogen and one $2 p$ atom added. The effects of spin polarization were tested to be unimportant here, because all electronic states do efficiently hybridize into a common metallic-type ban with low state density at the Fermi level, and no localized magnetic states are formed in none of the systems studied.

The 48-Ti supercell is shown in Fig. 1 within an (orthorhombic) box on which periodic boundary conditions are imposed. The octahedral interstitial, occupied by a $2 p$ atom, is shown in the left bottom corner by a black sphere. Tetragonal interstitials in different settings with respect to it are shown in the right panel of Fig. 1 as light red spheres. The "e" position is the most distant one in the supercell chosen, taking into account periodic boundary conditions. In each geometry ("a" to "e" positions of atomar $\mathrm{H}$ relative to $\mathrm{C}, \mathrm{N}, \mathrm{B}$ impurities), full structure relaxation has been allowed.

We analyzed the total energies in five different arrangements of hydrogen around each of $2 p$ dopants, with full lattice relaxation taken into account in each case, as a measure of "interaction potential" between $\mathrm{B} / \mathrm{C} / \mathrm{N}$, on one side, and $\mathrm{H}$, on the other side; the results are shown in Fig. 2. The "e" position represents the "infinite" separation of hydrogen from the $2 p$ atom; it is seen that a more close placement of hydrogen to $\mathrm{C}$ or $\mathrm{N}$ results in a quite appreciable energy gain 


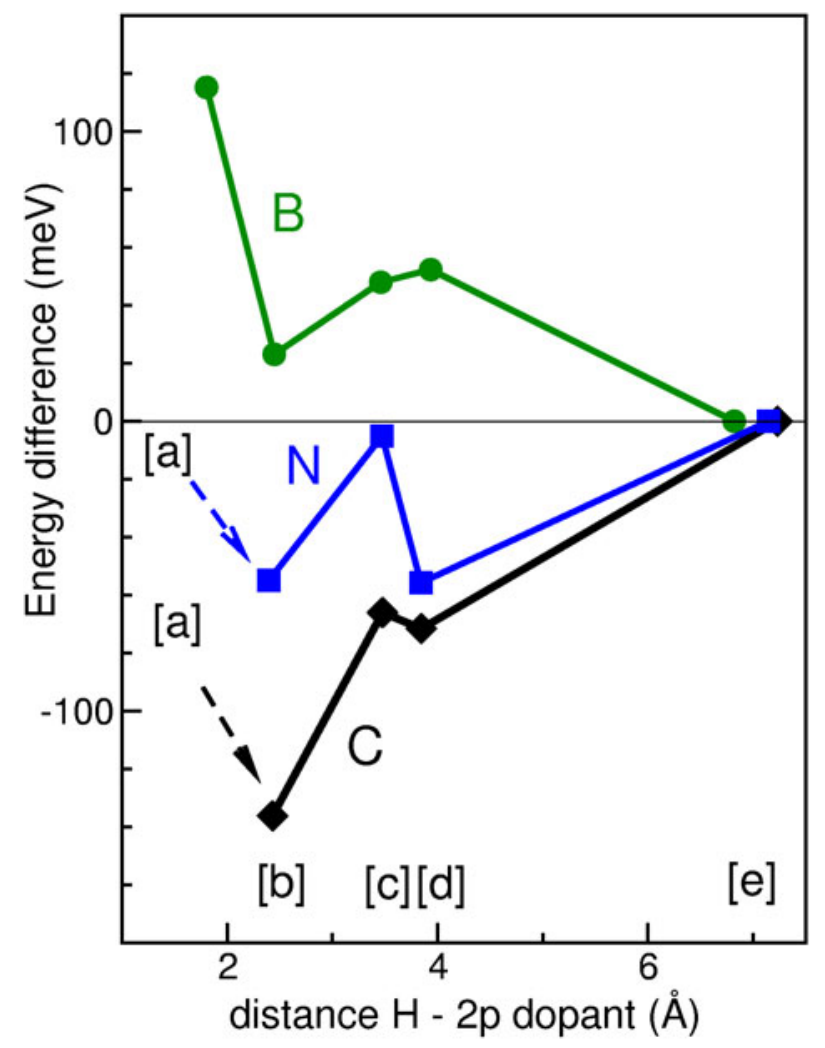

Figure 2: Calculated total energies, relative to that in the "e" configuration, for different placements of the (tetra)-interstitial $\mathrm{H}$ relative to (octa)-interstitial B, $\mathrm{N}$, or $\mathrm{C}$. The "a" configuration of $\mathrm{H}$ near the $\mathrm{N}$ or $\mathrm{C}$ site is unstable; the $\mathrm{H}$ atom invariably migrates, in the course of sructure relaxation, through the $\mathrm{Ti}_{3}$ face into the adjacent "b" position (see Fig. 1).

of some tens of meV (10 meV corresponds to $11.6 \mathrm{~K}$,or $0.23 \mathrm{kcal} / \mathrm{mol})$. An N or C interstitial impurity in titanium creates a large region in which the hydrogen affinity of titanium metal is substantially increased, which may explain the observed enhancement of its sorption capability. It is noteworthy that the $\mathrm{C}-\mathrm{H}$ or $\mathrm{N}-\mathrm{H}$ "interaction potentials" are not smooth but reveal a clear tendency to trap hydrogen in "b" and "d" positions, rather than in "a" or "c". In fact we failed to stabilize hydrogen in the "a" position close to carbon or nitrogen - it invariably diffuses into the neighbouring " $b$ " interstitial. It is seen from Fig. 1 that in the preferencial settings " $b$ " and "d", the hydrogen is bonded to a $2 p$ atom via Ti bridges, a single intermediate Ti atom (in "d") or a Ti pair, almost in-plane with $\mathrm{H}$ and the $2 p$ atom (in "b"). These configurations favour an efficient hybridization of $\mathrm{H} 1 s$ and $\mathrm{C}, \mathrm{N} 2 p$ states with Ti3 $d$ and gain in the binding energy. The local density of states (DOS) shown in Fig. 3 confirms this analysis. Indeed, the $2 p$ DOS of "N close to $\mathrm{H}$ " reveals a bonding peak at the position of $\mathrm{H} 1 s$, as it does the $3 d$ DOS of "Ti close to H". On the contrary, the hydrogen "close to B" in the right panel of Fig. 3 develops a splitting of its $1 s$ peak into two, avoiding hybridization with $\mathrm{B} 2 p$. A detailed analysis of involved molecular orbitals will be given elsewhere. It is seen from Fig. 2 that the placement of $\mathrm{H}$ in the vicinity of boron costs energy; however, all hydrogen placements "a" through " $\mathrm{e}$ " around boron are metastable, without the abovementioned effect of hydrogen squeezed out of the "a" site .

Summarizing, we simulated the effect of interstitial C, N, and B on the hydrogen affinity of metallic $\alpha$-titanium. The above dopants seems to be those which enter the titanium bulk in the process of milling the titanium with graphite, or boron nitride. We found that the addition of carbon, or (to a slightly less extent) of nitrogen does indeed improve the bonding of hydrogen in Ti lattice; not less important, this effect is quite long-ranged and extends over many tetrahedral sites up to $\sim 4-5 \AA$ around the $\mathrm{C}$ or $\mathrm{N}$ dopant. The effect of interstitial boron on the hydrogen 

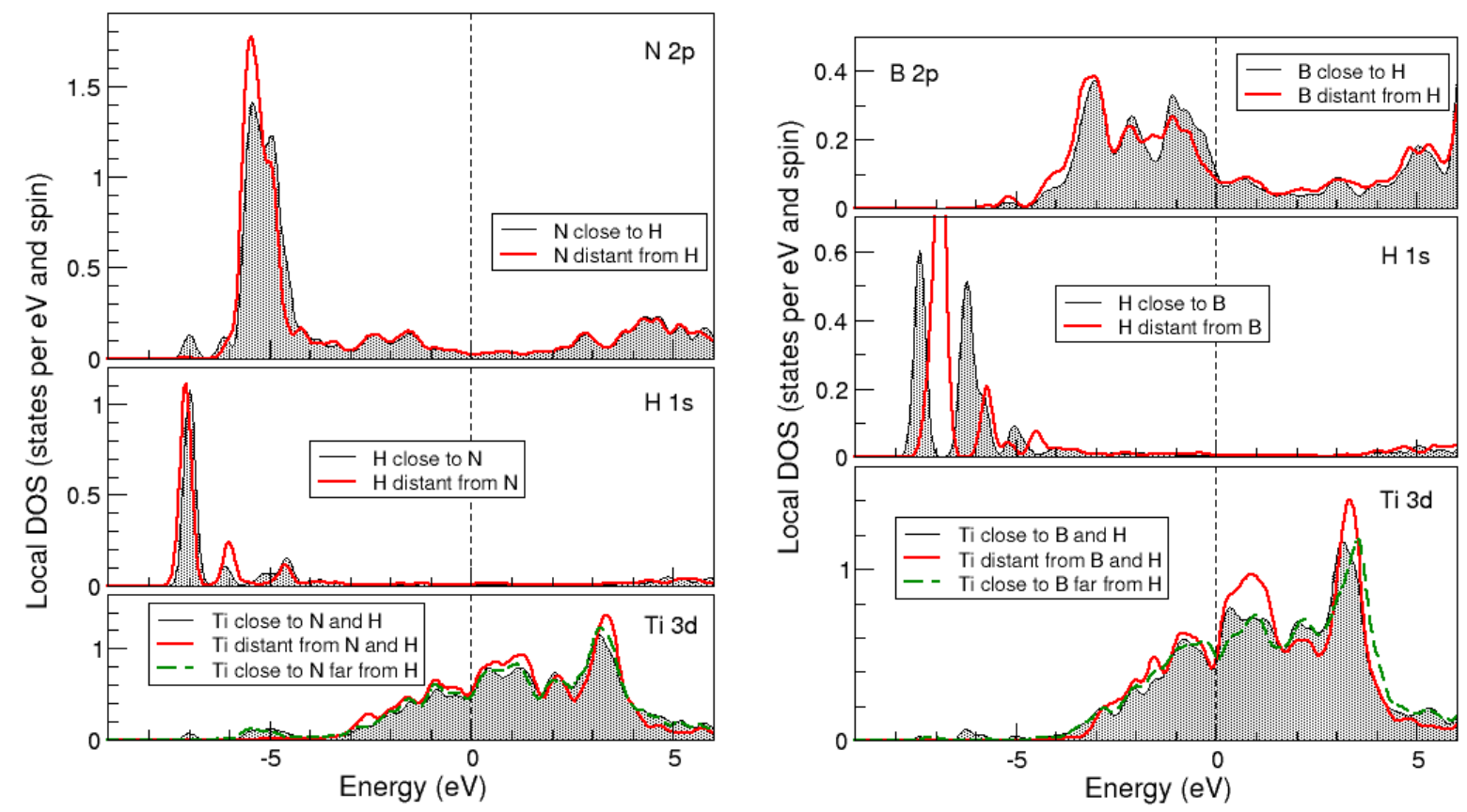

Figure 3: local densities of states of $2 p$ atom (left panel: $\mathrm{N}$, right panel: $\mathrm{B}$ ), $\mathrm{H}$, and Ti, for their different placements. "H close to N/B" means the "b" configuration of Fig. 1. Zero energy corresponds to the Fermi level.

absorption seems to be negative, but relatively small; it can be implied from experiment, however, that the elemental boron does not enter the Ti bulk as efficiently as carbon or nitrogen do. Taken together, this explains, from the microscopic viewpoint, an enhancement of hydrogen sorption in Ti under milling with either graphite, or boron nitride.

The work was supported by INTAS (project No 05-1000005-7672). We gratefully acknowledge the Russian Academy of Sciences Program (Project 01.2.006 13395). This work is also partly supported by the Russian Science Foundation for Basic Research (RFBR projects 07-0300610 and 08-02-00148), the Natural Sciences and Engineering Research Council of Canada (NSERC) and the Canada Research Chair program.

1. E.Z. Kurmaev, O.S. Morozova, T.I. Khomenko, Ch. Borchers, S.N. Nemnonov, Y. Harada, T. Tokushima, H. Osawa, T. Takeuchi and S. Shin, J. Alloys and Comp. 395, 240 (2005).

2. C. Borchers, O.S. Morozova, T.I. Khomenko, A.V. Leonov, A.V. Postnikov, E.Z. Kurmaev, A. Moewes and A. Pundt, J. Phys. Chem. B (submitted).

3. J.M. Soler, E. Artacho, J.D. Gale, A. García, J. Junquera, P. Ordejón and D. Sánchez-Portal, J. Phys.: Condens. Matter 14, 2745 (2002).

4. http://www.uam.es/siesta

5. N. Troullier and J.L. Martins, Phys. Rev. B 43, 1993 (1991). 